\title{
Interference of Enalaprilat on Glomerular Permeability to Macromolecules (IgG) in Acute Unilateral Ureteral Obstruction in Rats
}

\author{
Rene M. Oliveira, Antonio A. Oliveira Neto, Luiz C. Miranda \\ Section of Urology, Clementino Fraga Filho University Hospital, UFRJ, Rio de Janeiro, RJ, Brazil
}

\begin{abstract}
Introduction: Unilateral ureteral obstruction breaks out events that cause the transitory increase of glomerular permeability to macromolecules, both in the obstructed kidney and in the contralateral kidney, suggesting the presence of some factor, with a systemic action, liberated as a response to the obstruction. We know that the rennin-angiotensin system is activated by acute ureteral obstruction. We have developed an experiment to assess the role of angiotensin II on the glomerular permeability to $\operatorname{IgG}$ due to acute ureteral obstruction, using enalaprilat, an angiotensin enzyme conversion inhibitor, to block the effects of the activation of the rennin-angiotensin system.

Materials and Methods: We have used 45 adult Wistar female rats, distributed into 3 main groups: a control group with 5 animals and 2 experiment groups each one with 10 animals submitted to unilateral ureteral obstruction and nephrectomy at 60 and 120 minutes. Each experiment group had its simulation correspondent (sham). We have studied both kidneys through the direct immunofluorescence method.

Results: We have found positive permeation in animals without enalaprilat in both kidneys and negative permeation in those in which the drug was used.

Conclusion: We have concluded that enalaprilat interferes in this alteration of permeability, suggesting that angiotensin II is involved in the loss of selectivity of the glomerular membrane.
\end{abstract}

Key words: ureteral obstruction; immunoglobulin G; angiotensin II; enalaprilat; immunofluorescence

Int Braz J Urol. 2006; 32: 216-21

\section{INTRODUCTION}

Acute ureteral obstructions a common situation in urological practice. The obstructed kidney suffers both rough morphologic alterations and microscopic ones, which were demonstrated in experimental studies and through the study of surgical specimens (1-4).

In a line of research on the alterations of glomerular permeability to macromolecules in an experi- mental situation of acute unilateral ureteral obstruction, Henriques (5) demonstrated the permeation of rat endogenous IgG through the glomerular mesangium of the obstructed kidney. Interestingly enough, he also observed a similar phenomenon in contralateral kidney, without blockage to the urinary flow, in more intensity between 60 and 120 minutes after the obstruction.

This alteration of selectivity of the glomerular blockage on the contralateral kidney makes 
us suppose the action of some factor that has been liberated by the acute ureteral obstruction and that has a systemic action.

Acute ureteral obstruction is a potent activator of the rennin-angiotensin system that once activated is capable of promoting systemic actions (610). Based on this information, we have developed an experiment using acute unilateral obstruction as a factor to activate the rennin-angiotensin system, both to observe the selectivity alteration of the permeability of the glomerular filtration blockage after this activation and to verify the responsibility of angiotensin II in the phenomenon of the alteration of the selective permeability of the glomerular filtration membrane.

To test our hypothesis we have used an angiotensin I enzyme conversion inhibitor for angiotensin II as a blocker to the events broken out by the activation of the rennin-angiotensin system. We have proceeded to the histopathological analysis, through the direct immunofluorescence technique, both of the obstructed kidney and of the kidney without obstruction.

\section{MATERIALS AND METHODS}

We used forty-five Wistar albino female rats with weights varying from 240 to $300 \mathrm{~g}(255.55 \pm 9.35$ g.) and aged 8 months. Animals were kept in the animal room of the Experimental Lab, in individual cages, were fed with industrialized food used by the animal room and water ad libitum. At the day of the surgery, they were on a fast of solid foods. The weight of each animal was checked before surgery. The experimental protocol was approved by the animal research ethics commission.

Animals were divided into 3 groups: Group 1 - control (5 animals), Group 2 - ureteral ligature (10 animals) and Group 3 - enalaprilat previous to the ureteral ligature (10 animals). All animals were submitted to bilateral nephrectomy. On groups 2 and 3 , nephrectomy was accomplished in half of the animals at 60 minutes and on the other half at $120 \mathrm{~min}$ utes. Groups 2 and 3 have simulation correspondents (sham).
The enalaprilat was the drug used in the experiment, in the form of injectable solution. The dosage used was $1 \mathrm{mg} / \mathrm{kg}$, adequate for the obtainment of the angiotensin enzyme conversion inhibitor effect in animals (11). In those animals in which enalaprilat was used its administration was made by injection in the inferior vena cava 15 minutes before the ureteral ligature, time enough for the distribution of the drug and the obtainment of the angiotensin enzyme conversion inhibitor effect (11). For sham groups we have administered distilled water.

We have used sevoflurane as inhaling anesthetic agent, administered by means of a vaporizing device for small animals anesthesia developed by the experimental lab.

The animals were submitted to median laparotomy, we have identified the left ureter using an optic magnification with a $2.5 \mathrm{X}$ surgical magnifier, and we performed the complete ureteral occlusion in its upper $1 / 3$ with cotton 3-0 suture.

Once the ureteral occlusion time set forth in this work's protocol was finished, we performed the bilateral nephrectomy and the animals, still under anesthesia were transposed to a glass shade saturated with sulphuric ether for the euthanasia to be performed.

All surgical specimens at the moment of their extraction were longitudinally cleavage, involved in aluminum paper, identified by group, animal number and side (left or right) and stored in a liquid nitrogen container.

For direct immunofluorescence reaction, we use antiserum for rat IgG (sheep anti rat IgG:FITC AAR10F).

Histopathologic analysis started after the obtainment of all surgical specimens with the application of the direct immunofluorescence technique, in histological sections of preserved tissue. Group after group the material was prepared and observed in a Zeiss microscope. The elapsed time between the preparation and the reading of each group of slides took no longer than 2 hours, and all histopathological analysis was performed in 4 days. The processed material corresponded to the totality of each kidney, since the organs were not larger than $2 \mathrm{~cm}$ in the major axis. We proceeded the observation of all glomeruli and the results were collected from this analysis. 
In histopathological analysis, we considered positive mesangial staining when we observed a green brilliant coloration highlighting the glomeruli under the microscope ultraviolet light, and negative when this staining was continuous with background coloration or inexistent.

The most representative fields were selected for illustration with image registration by NikonCoolpix de 3.44 megapixels adapted to the microscope with automatic saturation, brightness and contrast adjustment and exposure time of $1 / 2$ second.

Statistical analysis was performed by Fisher exact test to compare positivity among studied groups. The criterion of significance is 5\%. Statistical analysis was processed by the statistical software SAS ${ }^{\circledast}$ System.

\section{RESULTS}

In the control group the mesangial staining was negative.

All 10 animals of group 2 presented positive mesangial staining, and one animal of the sham group also presented positive staining. We have observed that the proportion of positive results in group 2 was significantly larger than in the sham group ( $\mathrm{p}<$ 0.0001).

All animals of group 3 presented negative mesangial staining but one animal of the sham group presented positive mesangial staining. Statistical analysis demonstrated that there was no significant difference between the 2 groups $(p=0.50)$.

Comparison between group 2 and 3 showed a significant difference with $\mathrm{p}<0.0001$.

On Figure-1 we present the photomicrography of a glomerulus with positive mesangial staining.

\section{COMMENTS}

It is a consensus among investigators that, in physiologic conditions, macromolecules the size of IgG (molecular radius of approximately $55 \AA$, molecular weight of 150.000 Daltons) are not found in significant quantities in the glomerular filter but can be detected in the mesangium. There is, however, a physiological transit of macromolecules through the vascular endothelium fenestrations of the glomerular tuft, but are retained by the basal membrane and by the filtration diaphragm and come back to the intravascular space, via the lymphatics, or they are metabolized by mesangial cells without immunological reaction involved in this process (12-16).

Acute ureteral unilateral obstruction causes alterations in glomerular perfusion and in the glomerular filtration rate. Those alterations act as activators of the rennin-angiotensin system, with the increase in the secretion of rennin through the obstructed kidney, be it by a reduction in the concentration of sodium in the distal tubules, be it by baroreceptors distension localized in the afferent arteriole. $(1,3,4)$.

The hypothesis is that the phenomenon of IgG endogenous permeability by glomerular filtration barrier of the animals submitted to acute unilateral ureteral obstruction, observed both in the obstructed kidney and in the contralateral kidney is related to the effects promoted by endogenous angiotensin II. Liberated from the activation of the rennin-angiotensin system broke out by the acute ureteral obstruction.

Alterations of glomerular filtration during unilateral ureteral obstruction were already described from the 5 to 8 minutes occlusion, in the affected kidney (3). In experimental work of unilateral ureteral

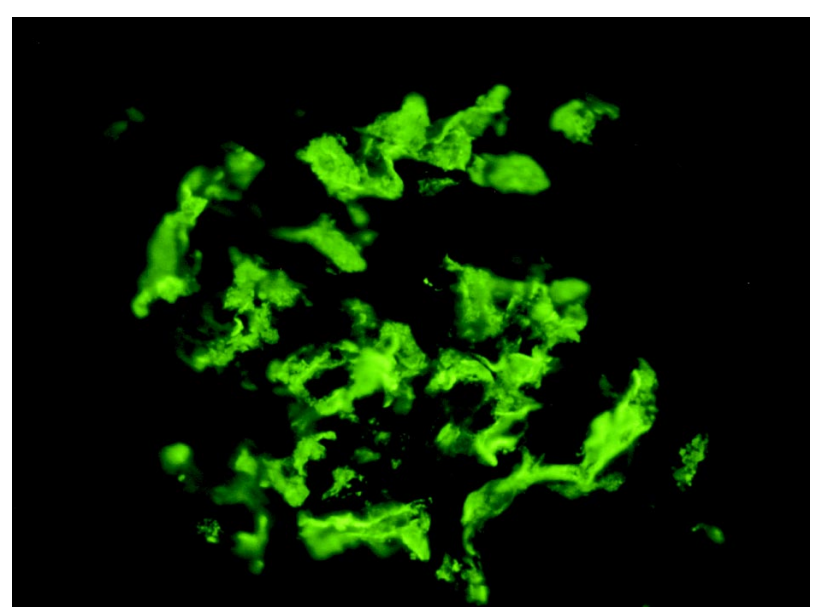

Figure 1 - Histological study showing positive glomerular staining (direct immunofluorescence, X400). 
obstruction, Henriques (5) demonstrated the passage of macromolecules ( $\mathrm{IgG}$ ) for the mesangium, a precocious and transitory phenomenon that is most intensively manifested between 60 and 120 minutes after the installation of the unilateral ureteral obstruction, and is reduced after 120 minutes of obstruction, but was observed in both kidneys. However, it seemed to be valid to focus our study in this period of time. This is a model that makes more intense the mesangial transit of macromolecules, a phenomenon that can be easily demonstrated by the technique of direct immunofluorescence but does not involve immunologic processes.

We have observed that pertinent literature is poor in relation to this experiment, for, in general, only the obstructed kidney is analyzed. In this work we have considered only the qualitative aspect of the event for we supposed that angiotensin II participates in the alteration of the glomerular filtration barrier selectivity. We did not try to determine the intensity of this action, or if other substances liberated in response to the ureteral obstruction can alter this selectivity. We have made a ureteral obstruction and kidney extraction in subgroups of 60 and 120 minutes after the ureteral obstruction, for we do not have data on the time interval necessary to occur the probable action of the angiotensin II over the glomerular selectivity.

To test our hypothesis that the alteration of the glomerular permeability is related to the action of the angiotensin II, we use the pharmacologic obstruction of the angiotensin conversion enzyme, through the injection of enalaprilat before we performed a ureteral obstruction.

The study of the control group demonstrates that in a normal situation the mesangial transit of endogenous $\mathrm{IgG}$ is not detectable through the direct immunofluorescence technique since there was no positive staining in the kidney of those animals.

The study of the animals of group 2 evidences the intense permeability of IgG through the glomerular mesangium, demonstrated by the positive staining of both kidneys.

This mesangial transit can be influenced by the capilar hydrostatic pressure, by the molecular weight and protein electrical charge and by factors related to the mesangial matrix selectivity properties that can be altered as demonstrated in experimental situations, with the infusion of angiotensin II $(1,13,14,17)$.

When we observe the animals of group 2 (sham), we observe that one of the animals sacrificed at 60 minutes presented a positive mesangial staining. This data was not statistically significant, however, we can consider that factors activated by surgical procedure, unchained by the neuroendocrine reaction of the organic trauma, or other humoral mediators involved in the response to the acute unilateral ureteral obstruction, such as aracdonic acid metabolites (eicosanoids), the atrial natriuretic peptide, nitric oxide, endotelin, plaquetary activation factor, clusterin and the growth and transformation factor $\beta$ can also interfere in the glomerular filtration barrier permselectivity (3). We have made this registration as a possibility to develop other experiments in the same line of research.

The analysis of group 3 demonstrated that the use of an angiotensin enzyme conversion inhibitor interfered in the IgG mesangial transit, for we did not observe positive staining in one of the animals of group 3 (sham), sacrificed at 60 minutes of obstruction, that reinforces considerations over other factors involved in the alteration of the glomerular permeability even though this data does not have any statistical significance to this study.

The alteration of selectivity of the glomerular filtration membrane in the obstructed kidney was previously described $(13,17,18)$, however, the observation of the change in the glomerular filtration permselectivity in the contralateral kidney, observed in this work and previously reported $(5,19)$, makes us suppose the action of some factor that has a systemic action affecting such selectivity at distance, relating the loss of the glomerular filtration barrier selectivity with the increase of the mesangial transit of macromolecules in situation of activation of the rennin-angiotensin system.

When we compare groups 2 and 3, we find a significant difference that points out to the validity of the experiment to demonstrate the increase of the IgG mesangial transit in the ureteral obstruction with positive staining in both kidneys of the ani- 
mals of group 2, and the interference caused by the obstruction of the angiotensin conversion enzyme with negative staining in the kidneys of the animals of group 3 .

Our interpretation thus, is that there are statistical evidences of the interference of enalaprilat over the permeability of $\mathrm{IgG}$ in the acute unilateral ureteral obstruction.

\section{CONCLUSIONS}

We concluded that the acute unilateral ureteral obstruction in the rat, causes alteration of the glomerular selective filtration in the kidney free of obstruction, allowing the permeability of endogenous IgG.

There are evidences that the use of enalaprilat presents interference in the endogenous IgG permeability by the glomerular filtration membrane of the kidney free of obstruction in the rat.

Angiotensin II, one of the humoral factors liberated by the acute ureteral obstruction is related to the glomerular filtration membrane permselectivity_of the kidney free of obstruction in the rat, observed between 60 and 120 minutes of ureteral obstruction.

\section{CONFLICT OF INTEREST}

None declared.

\section{REFERENCES}

1. D’Amico G, Bazzi C: Pathophysiology of proteinuria. Kidney Int. 2003; 63: 809-25.

2. Nangaku M: Final common pathways of progression of renal diseases. Clin Exp Nephrol. 2002; 6: 182-9.

3. Gulmi FA, Felse D, Vaugham ED Jr: Pathophysiology of Urinary Tract Obstruction. In: Walsh PC (ed.), Campbell's Urology. Philadelphia, Saunders. 2002; pp. 411-62.

4. Wen JG, Frokiaer J, Jorgensen TM, Djurhuus JC: Obstructive nephropathy: an update of the experimental research. Urol Res. 1999; 27: 29-39.
5. Henriques FP: Immunoglobulin Absorption by the Glomerular Mesangium in Rats with Ureteral Obstruction. Thesis (Doctoral Degree in Surgery). Federal University of Rio de Janeiro, UFRJ, Brazil, 1998. [in Portuguese]

6. Frokiaer J, Pedersen EB, Knudsen L, Djurhuus JC: The impact of total unilateral ureteral obstruction on intrarenal angiotensin II production in the polycalyceal pig kidney. Scand J Urol Nephrol. 1992; 26: 289-95.

7. Frokiaer J, Knudsen L, Nielsen AS, Pedersen EB, Djurhuus JC: Enhanced intrarenal angiotensin II generation in response to obstruction of the pig ureter. Am J Physiol. 1992; 263: F527-33.

8. Klahr S, Ishidoya S, Morrissey J: Role of angiotensin II in the tubulointerstitial fibrosis of obstructive nephropathy. Am J Kidney Dis. 1995; 26: 141-6.

9. Oliw E: Acute unilateral ureteral occlusion increases plasma renin activity and contralateral urinary prostaglandin excretion in rabbits. Eur J Pharmacol. 1978; 53: 95-102.

10. Pimentel JL Jr, Martinez-Maldonado M, Wilcox JN, Wang S, Luo C: Regulation of renin-angiotensin system in unilateral ureteral obstruction. Kidney Int. 1993; 44: 390-400.

11. Tocco DJ, deLuna FA, Duncan AE, Vassil TC, Ulm EH: The physiological disposition and metabolism of enalapril maleate in laboratory animals. Drug Metab Dispos. 1982; 10: 15-9.

12. Deen WM, Lazzara MJ, Myers BD: Structural determinants of glomerular permeability. Am J Physiol Renal Physiol. 2001; 281: F579-96.

13. Keane WF, Raij L: Relationship among altered glomerular barrier permselectivity, angiotensin II, and mesangial uptake of macromolecules. Lab Invest. 1985; 52: 599-604.

14. Michael AF, Keane WF, Raij L, Vernier RL, Mauer SM: The glomerular mesangium. Kidney Int. 1980; 17: 141-54.

15. Olivetti G, Kithier K, Giacomelli F, Wiener J: Glomerular permeability to endogenous proteins in the rat: effects of acute hypertension. Lab Invest. 1981; 44: 127-37.

16. Raij L, Keane WF: Glomerular mesangium: its function and relationship to angiotensin II. Am J Med. 1985; 79: 24-30.

17. Lapinski R, Perico N, Remuzzi A, Sangalli F, Benigni A, Remuzzi G: Angiotensin II modulates glomerular capillary permselectivity in rat isolated perfused kidney. J Am Soc Nephrol. 1996; 7: 653-60. 


\section{$\lg \mathrm{G}$ in Ureteral Obstruction in Rats}

18. Mayer G, Lafayette RA, Oliver J, Deen WM, Myers BD, Meyer TW: Effects of angiotensin II receptor blockade on remnant glomerular permselectivity. Kidney Int. 1993; 43: 346-53.
19. Miranda LC: Behavior of Immunoglobulin $\mathrm{G}$ and $\mathrm{M}$ in the Renal Ischemia Reperfusion in Rats. Immunofluorescence Study. Thesis (Doctoral Degree in Surgery). Federal University of Rio de Janeiro, UFRJ, Brazil, 1992; p. 91. [in Portuguese]

Accepted after Revision:

January 5, 2006

\footnotetext{
Correspondence address:

Dr. René Murilo de Oliveira

Rua Conde de Irajá, 97/404 - Botafogo

Rio de Janeiro, RJ, 22 271-020, Brazil

E-mail: renemurilo@aol.com
} 